\title{
Isolated thrombosis of right spermatic vein with underlying Factor V Leiden mutation
}

\author{
Deniz Bolat, MD;1 Bulent Gunlusoy, MD;' Serkan Yarimoglu, MD;i Funda Ozsinan, MD;² Serife Solmaz, MD; ${ }^{3}$ \\ Fatma Gul Imamoglu, MD2
}

'Bozyaka Training and Research Hospital, Department of Urology, Izmir, Turkey; ${ }^{2 B o z y a k a ~ T r a i n i n g ~ a n d ~ R e s e a r c h ~ H o s p i t a l, ~ D e p a r t m e n t ~ o f ~ R a d i o l o g y, ~ I z m i r, ~ T u r k e y ; ~}{ }^{3 B o z y a k a ~ T r a i n i n g ~ a n d ~ R e s e a r c h ~ H o s p i t a l, ~}$ Department of Hematology, Izmir, Turkey

Cite as: Can Urol Assoc J 2016;10(9-10):E324-7. http://dx.doi.org/10.5489/cuai.3208 Published online September 13, 2016

\section{Abstract}

Spermatic vein thrombosis is a very uncommon clinical entity. Most cases involve the left side. Herein, we present an unusual case of a young man who presented with spermatic vein thrombosis on the right side with an underlying Factor $\mathrm{V}$ Leiden mutation. To our knowledge, it is the first case in the literature.

\section{Introduction}

Spermatic vein thrombosis is a rare event and requires careful examination for the differential diagnosis. Clinical presentation is usually with left acute testicular pain and testicular swelling in both children and adults. ${ }^{1}$ Most cases involved the left side. ${ }^{2}$ There are a limited number of patients, with only 18 cases of spontaneous vein thrombosis noted in the literature. ${ }^{1}$ We present a case of isolated spermatic vein thrombosis in the right spermatic vein with right varicocele of the testis and discuss treatment alternatives in light of current literature.

\section{Case report}

A 35- year-old male with right testicular pain and swelling was admitted to the outpatient clinic. Although medical and surgical history of the patient was unremarkable, he did not express any etiologic factors, such as known trauma, severe exercise, alcohol consumption, or smoking. The physical examination of testes revealed right Grade II varicocele with normal-sized testicles. Scrotal Doppler ultrasound demonstrated a $3 \mathrm{~cm}$ in length and $6 \mathrm{~mm}$ in thickness heterogeneous, tubular cystic mass in the right spermatic vein accompanying Grade II varicocele on the same side (Fig. 1). There was no bloodflow at Doppler imaging (Fig. 2). The findings were considered as spermatic vein thrombus in the initial diagnosis.
Magnetic resonance imaging of the abdomen and pelvis demonstrated dilated, thick-walled, thrombosed testicular vein with increased intraluminal signaling in the right spermatic cord in axial T1A-weighted fat-suppressed images, with normal other organ findings (Fig. 3).

Basic laboratory tests, including coagulation parameters, were normal. The patient consulted with the hematology department to assess potential reasons for this clinical entity. Complete laboratory workup (erythrocyte sedimentation rate, C-reactive protein, antithrombin III, prothrombin gene, protein $\mathrm{C}$, protein $\mathrm{S}$, rheumatoid factor, anti-nuclear antibody, anti-phospholipid, lupus anticoagulant, anti-cardiolipin antibodies, and homocysteine level) was negative, except for Factor $\mathrm{V}$ Leiden mutation heterozygous positivity. Anticoagulant therapy was started with subcutaneous enoxaparine and oral warfarin. Enoxaparine was stopped once the patient's international normalized ration reached level 2-3

During the followup period, the patient's testicular pain was completely relieved after a month of the anticoagulant therapy. Control scrotal Doppler ultrasound on the third month showed incomplete resolution of the thrombosis 1 $\mathrm{cm}$ in length and $3.5 \mathrm{~mm}$ in thickness in the right spermatic vein (Fig. 4).

\section{Discussion}

Spermatic vein thrombosis is an unexpected finding in the differential diagnosis of acutely painful scrotum. ${ }^{3}$ Most of these cases are managed surgically as if they had an incarcerated inguinal hernia. ${ }^{4}$ Additionally, epididymitis, spermatic cord disease (such as tortion), or benign and malignant tumours of spermatic cord should be kept in mind in the differential diagnosis. ${ }^{3-6}$ Testicular pain and swelling are the most common symptoms. ${ }^{1}$ Spermatic vein thrombosis is almost always found on the left side. ${ }^{1}$ In the physical examination, our patient had mild pain and dilated palpable spermatic vein suggesting right varicocele. Leaving aside the vein thrombosis, right varicocele is an important clinical sign to do detailed research at the renal hilus level or in the 


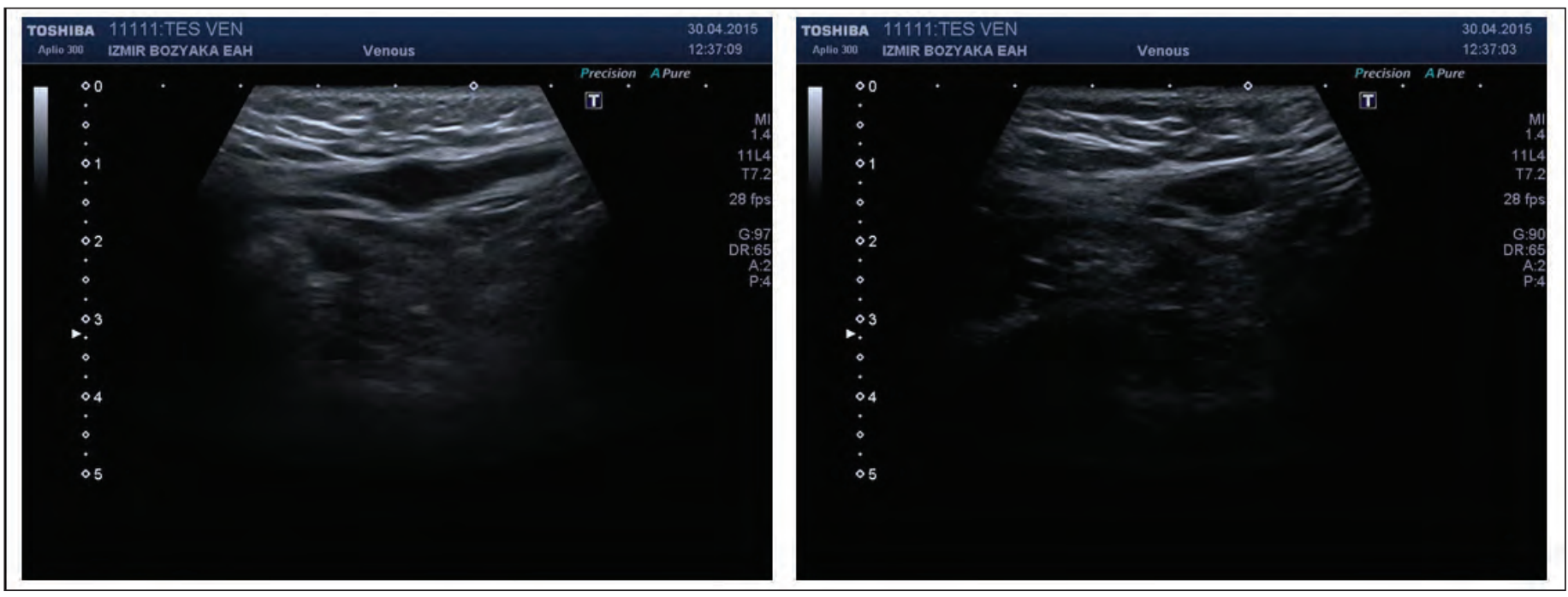

Fig. 1. Grey scale ultrasonography demonstrated non-compressed thrombosed tubular venous structure with increased wall thickness and focal diameter increase within the right spermatic cord. Within this tubular structure, focal echoes that belong to thrombosis can be seen with an antero-posterior diameter 6 mm.

retroperitoneal region to rule out renal tumours with renal vein, vena cava thrombosis, or retroperitoneal tumours.

In the etiology of isolated spermatic vein thrombosis, there are many possible predisposing factors, such as trauma to the vascular endothelium, slow venous flow, and hypercoagulability. ${ }^{7}$ Kayes et al reported that spontaneous vein thrombosis could be related to prolonged vigorous sexual activity or sport activity, tumours of the genitourinary tract, infections, trauma, inguinal hernia surgery, long-hour flights, and the use of some drugs. ${ }^{8}$ Mirilas et al cautioned for a complex system that should be carefully considered when opting for the subinguinal approach and found it necessary to carry out a comprehensive preoperative hemodynamic assessment of the vein reflux to decide on the most suitable surgical technique. ${ }^{9}$
The Factor $V$ Leiden mutation and the prothrombin gene mutation are the two most common known genetic mutations that predispose to venous thromboembolism (VTE). ${ }^{10,11}$ These mutations account for $20 \%$ of first VTE episodes. The risk of VTE increases 3-8-fold in heterozygous Factor $V$ Leiden mutation-positive patients. ${ }^{12}$ In case of idiopathic VTE with hereditary components, long-term anticoagulant therapy is mandatory to prevent the recurrent VTEs. ${ }^{13}$

Among the acceptable treatment strategies for isolated spermatic vein thrombosis, conservative approaches are reasonable. It is also possible to manage the patient surgically. Hashimoto and Vibeto reported that there is no need to excise the thrombosed plexus, as evidenced by the good results in their case. ${ }^{2}$ We believe that beginning treatment conservatively is more logical than surgery. By this

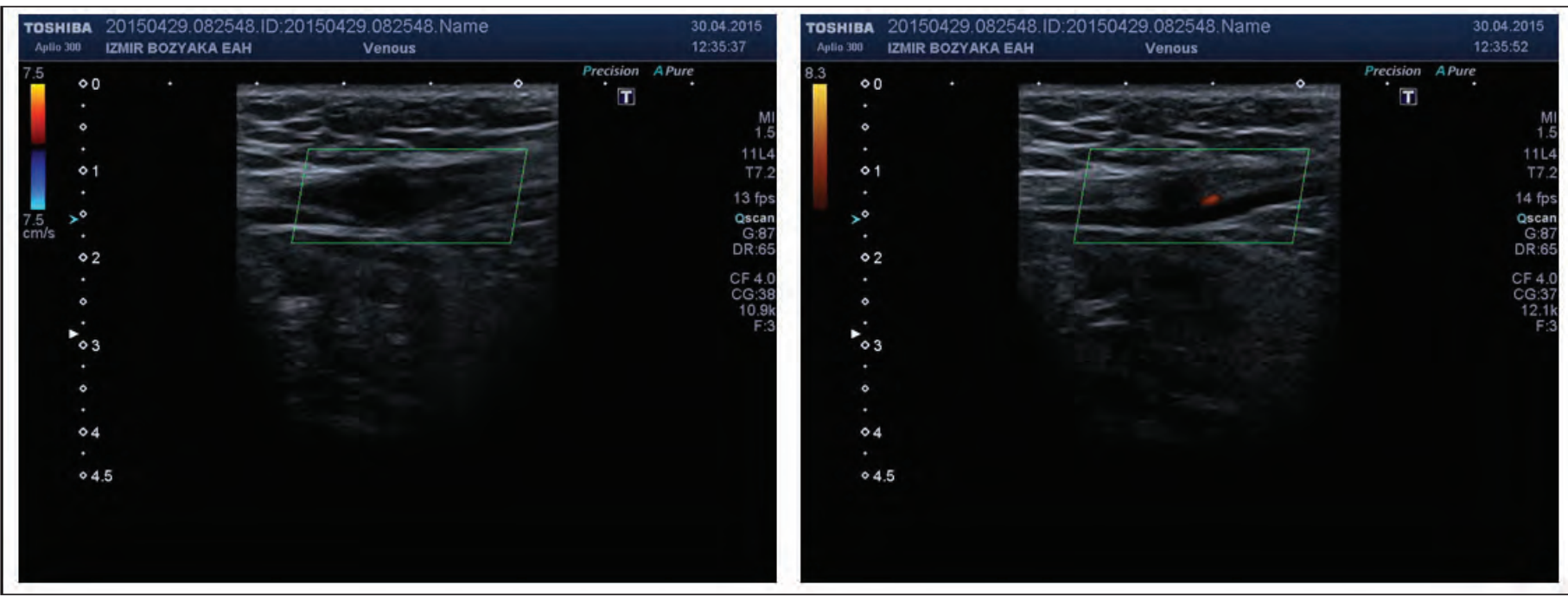

Fig. 2. On colour Doppler ultrasound and power Doppler ultrasound, no filling with the colour was seen in the lumen of this vein within the right spermatic cord. On power Doppler ultrasound, filling was seen within the neighbouring arterial structure, but not within the vein. 


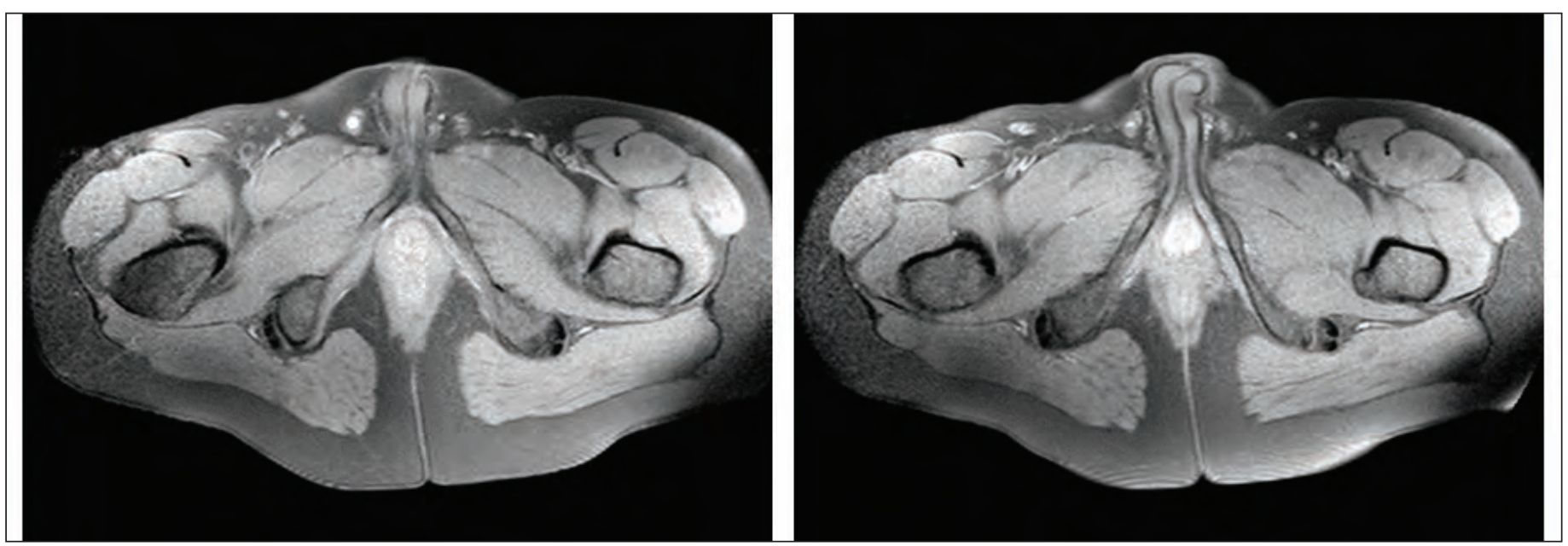

Fig. 3. Fat-compressed axial T1A magnetic resonance images demonstrated thrombosed tubular venous structure with increased wall thickness and focal diameter increase within the right spermatic cord. Within this venous structure, intraluminal signal intensity was increased.

way, a potential spread of coagulum can be eliminated. Anticoagulant therapy with bed rest and scrotal support can be used clinically.

\section{Conclusion}

Isolated spermatic vein thrombosis is a rare event. Although our case is right-sided, spermatic vein thrombosis is almost always found at the left side. If there is no other concomitant disease that necessitates urgent surgical intervention, beginning the treatment conservatively instead of excising the thrombosed segment is more suitable.
Competing interests: The authors report no competing personal or financial interests.

This paper has been peer-reviewed.

\section{References}

1. Castillo $0 \mathrm{~A}$, Diaz M, Vitagliano GJ, et al. Pulmonary thromboembolizm secondary to left spermatic vein thrombosis: A case report. Urol Int 2008;80:217-8. http://dx.doi.org/10.1159/000112617

2. Hashimoto L, Vibeto B. Spontaneous thrombosis of the pampiniform plexus. Scand I Urol Nephrol 2006;40:252-4. http://dx.doi.org/10.1080/00365590600589823

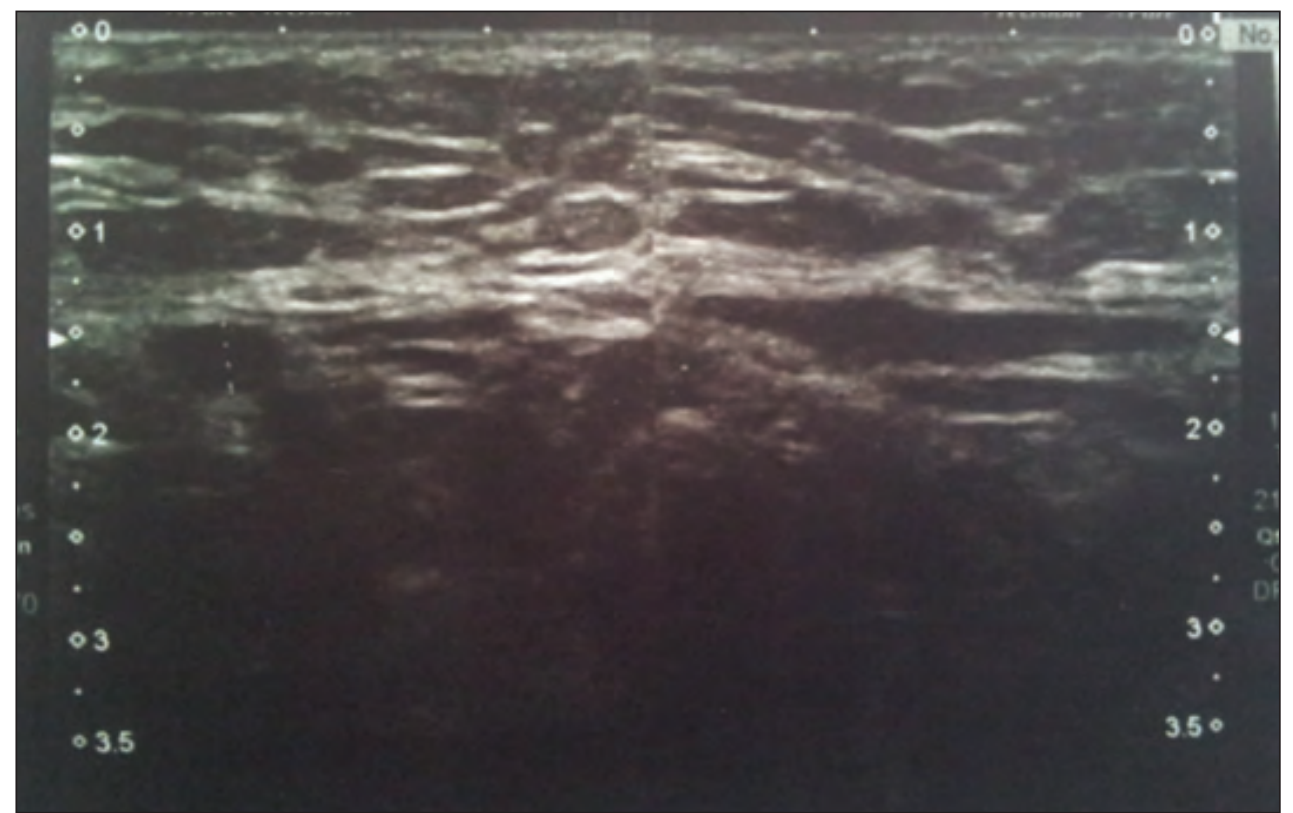

Fig. 4. On the third month control Doppler ultrasound, the regression was observed on the size of the thrombosis with an anterior-posterior diameter $3.5 \mathrm{~mm}$ and nearly full compression was revealed. 
3. Kleinclauss F, Della Negra E, Martin M, et al. Spontaneous thrombosis of left varicocele. Prog Urol 2001;11:95-6

4. Campagnola S, Flessati P, Fasoli L, et al. A rare case of acute scrotum. Thrombophlebitis from ectasia of the left pampiniforme plexus. Minerva Uroll Nephrol 1999:51:163-5.

5. Gleeson MJ, McDermott M, McDonald G, et al. Spontaneous thrombosis of the left spermatic vein. Br J Urol 1992; 70:567.

6. Kawabata G, Maeda H, Morisue K, et al. Spontaneous thrombosis of the pampiniform plexus-case report Nihon Hinyokika Gakkai Zasshi 1993;84:563-5. http://dx.doi.org/10.5980/ipnjurol1989.84.563

7. Zampieri N, Castellani R, Mantovani A, et al. Thromboses of the panpiniform plexi after subinguinal varicocelectomy. Pediatr Surg Int 2014;30:441-4. http://dx.doi.org/10.1007/s00383-013-3426-1

8. Kayes 0 , Patrick N, Sengupta A. A peculiar case of bilateral, spontaneous thromboses of the pampiniform plexi. Ann R Coll Surg Engl 2010;92:W22-3. http://dx.doi.org/10.1308/14787081 OX12822015504400

9. Mirilas $P$, Mentessidou A. Microsurgical subinguinal varicocelectomy in children, adolescents, and adults: Surgical anatomy and anatomically justified technique. J Androl 2012;3:338-49. http://dx.doi. org/10.2164/jandrol.111.013052

10. Goldhebar SZ. Venous thrombolism: Epidemiology and magnitude of the problem. Best Pract Res Clin Haematol 2012;25:235-42. http://dx.doi.org/10.1016/i.beha.2012.06.007
11. Kearon C. Influence hereditary or acquired thrombophilias on the treatment of venous thromboembolism. Curr Opin Hematol 2012;19:363-70 http://dx.doi.org/10.1097/MOH.0b013e328356745b

12. Marchiori A, Mosena L. The risk of recurrent venous thromboembolism among heterozygous carriers of factor V Leiden or prothrombin G20210A mutation. A systematic review of prospective studies. Haematologica 2007;92:1107-14 http://dx.doi.org/10.3324/haematol.10234

13. Prandoni $P$, Noventa $F$. The risk of recurrent venous thromboembolism after discontinuing anticoagulation in patients with acute proximal deep vein thrombosis or pulmonary embolism. A prospective cohort study in 1626 patients. Haematologica 2007; 92:199-205. http://dx.doi.org/10.3324/haematol.10516

Correspondence: Dr. Deniz Bolat, Department of Urology, Bozyaka Training and Research Hospital, Karabaglar, Izmir,Turkey; drbolał@hotmail.com 